\title{
Host specificity of Symbiodinium variants revealed by an ITS2 metahaplotype approach
}

\author{
Edward G Smith, Remi N Ketchum and John A Burt \\ Center for Genomics and Systems Biology, New York University Abu Dhabi, Abu Dhabi, UAE
}

\begin{abstract}
Analysis of the widely used ITS region is confounded by the presence of intragenomic variants (IGVs). In Symbiodinium, the algal symbionts of reef building corals, deep-sequencing analyses are used to characterise communities within corals, yet these analyses largely overlook IGVs. Here we consider that distinct ITS2 sequences could represent IGVs rather than distinct symbiont types and argue that symbionts can be distinguished by their proportional composition of IGVs, described as their ITS2 metahaplotype. Using our metahaplotype approach on Minimum Entropy Decomposition (MED) analysis of ITS2 sequences from the corals Acropora downingi, Cyphastrea microphthalma and Playgyra daedalea, we show the dominance of a single species-specific Symbiodinium $\mathrm{C} 3$ variant within each coral species. We confirm the presence of these species-specific symbionts using the psbA non-coding region. Our findings highlight the importance of accounting for IGVs in ITS2 analyses and demonstrate their capacity to resolve biological patterns that would otherwise be overlooked.

The ISME Journal (2017) 11, 1500-1503; doi:10.1038/ismej.2016.206; published online 17 February 2017
\end{abstract}

The taxonomy of diverse microbial groups (for example, fungi, green algae and dinoflagellates) is frequently distinguished using the ITS region of rDNA (LaJeunesse, 2001; Mai and Coleman, 1997; Schoch et al., 2012; Stern et al., 2012). However, there are multiple copies of rDNA present in genomes, with differing forms known as intragenomic variants (IGV). Such IGVs can confound ITSbased analyses (Álvarez and Wendel, 2003; Thornhill et al., 2007; Stat et al., 2011; Stern et al., 2012), but in the absence of more suitable markers, the ITS region remains a dominant means of characterising diverse microbial communities.

The ITS2 region underpins the taxonomy of the dinoflagellate Symbiodinium (LaJeunesse, 2005), a symbiont widely associated with cnidarians, most notably reef building corals. Symbiodinium is fundamental to the coral holobiont, contributing $>90 \%$ of the energy budget and influencing corals' capacity to respond to environmental stress (Muscatine et al., 1984; Berkelmans and Van Oppen 2006). Deep sequencing of Symbiodinium communities typically use the ITS2 region and are processed using OTU or phylotyping approaches (for example, Arif et al., 2014; Cunning et al., 2015). These approaches either incorporate IGVs within OTUs or do not consider

Correspondence: EG Smith, New York University Abu Dhabi, Centre for Genomics and Systems Biology, Saadiyat Island, Abu Dhabi 129188, UAE.

E-mail: edsmith@nyu.edu

Received 15 June 2016; revised 20 October 2016; accepted 25 November 2016; published online 17 February 2017 them in analyses. Instead of considering IGVs as incidental, here we show that the composition of ITS2 IGVs can be used to differentiate distinct taxonomic sub-groups, revealing biological patterns consistent with a highly polymorphic marker.

We compared symbiont communities among three coral species (Acropora downingii, Cyphastrea microphthalma and Platygyra deadalea) focusing on clade $\mathrm{C}$ as it is the most numerically dominant clade among these species at our sampling location (Hume et al., 2015; Supplementary Table 1). We assessed the Symbiodinium ITS2 sequence composition using an amplicon sequencing approach (see Supplementary Methods; $N=13-15$ ind./coral sp.). Briefly, amplification of ITS2 and psbA non-coding region (psbAncr) was performed with modified Sym_Var primers (Hume et al., 2015). Normalised libraries were then sequenced on the MiSeq Platform (Illumina) using the MiSeq v3 600-cycle kit. Demultiplexing, quality filtering, adapter removal, chimera removal and rarefaction (Supplementary Figure 1) were performed prior to analysis with the Minimum Entropy Decomposition (MED) pipeline (Eren et al., 2015). MED nodes were analysed using vegan (in R) and permutation multivariate analysis of variance (adonis in vegan). We compared our IGV approach with the OTU approach processed using the mothur pipeline for Symbiodinium (Schloss et al., 2009; Arif et al., 2014).

Symbiont communities from each species were initially analysed using the traditional OTU-based approach, with a 97\% cutoff; this showed the presence of a single OTU, identified as a C3-type 
symbiont, accounting for $100 \%$ of all ITS2 sequences. In essence, the OTU approach characterised all coral species as hosting the same host-generalist C3 symbiont type. As oligotyping (including minimum entropy decomposition, MED) has been shown to reveal biologically relevant patterns masked by OTU approaches (Eren et al., 2014,Eren et al., 2015), we then applied MED analyses to these symbiont communities. MED is ideally suited to analysing the extreme abundance of rare ITS2 variants in Symbiodinium (Arif et al., 2014) by identifying the biologically informative positions and therefore removing methodological and biological noise. Despite collapsing the data into distinct nodes, MED analyses retain the patterns observed in haplotype-based analyses (Supplementary Figures 2 and 3). In contrast to the OTU approach, MED analysis revealed 44 distinct MED nodes (sequences grouped together exclusively by informative positions), characterised by sequence variants of ITS2 types C3 and C3 Gulf (Supplementary Figure 4). The most abundant ITS2 nodes were common to all coral species, with the most abundant node accounting for $30-50 \%$ of ITS2 sequences in all three coral species. Although the relative proportion of nodes varied among species, they were remarkably consistent within species. Species-specific grouping was confirmed by multivariate analysis (Adonis, ITS2, $\mathrm{F}=213.52$, $r^{2}=0.918, P=0.000999$ ).

Without an additional marker, interpretation of these data is complex as each MED node could either represent a distinct symbiont or an intragenomic variant (IGV). There are two biologically distinct scenarios in which these data could be interpreted. These coral species could host a mixed community of symbionts (for example, Sampayo et al., 2007), kept in relatively consistent proportions within species, although the most abundant symbiont taxa would be shared across all coral species. Alternatively, each coral species could host a single symbiont taxon, which is distinguished only by the species-specific composition of its IGVs, herein referred to as an 'ITS2 metahaplotype'. Here we will describe these two scenarios using the terms 'mixed community' and 'single taxon' (an unresolved taxonomic unit), respectively.

In order to distinguish between these scenarios, we performed MED analysis on psbAncr, a marker that provides high phylogenetic resolution in Symbiodinium and although multi-copy, is typically present as a single dominant sequence with low abundances of IGVs (LaJeunesse and Thornhill, 2011). Under the mixed community scenario, we would predict a mixture of psbAncr MED nodes in each individual coral. For example, in $A$. downingi, we observed four abundant ITS2 MED nodes and if each node represents a separate symbiont, we should expect to see four correspondingly abundant psbAncr MED nodes. Furthermore, the abundant nodes should be shared across coral species. Our analyses shows this is not the case. Instead, each individual coral was dominated by a single psbAncr MED node, which is indicative of the presence of a single symbiont type (Figure 1d). In agreement with the ITS2 data, the psbAncr MED node composition results in speciesspecific clustering (Figures 1e and f-Adonis, psbAncr, $\left.\mathrm{F}=38.51, r^{2}=0.670, P=0.000999\right)$. Overall, these results support the single taxon scenario: that each coral species hosts a single speciesspecific symbiont characterised by its unique metahaplotype. These observations highlight the important role that IGVs could play in improving the taxonomic characterisation of Symbiodinium and other microbial groups, by providing insights into unique species-specific symbiont signatures that would have gone unnoticed by traditional ITS2 approaches.

Symbiodinium C3 was initially considered a widespread generalist (LaJeunesse, 2005). However, our observation of diverse variants, which are coral host species-specific, within C3 support previous work that shows that there is substantial cryptic diversity that is currently unresolved (Thornhill et al., 2014). This has important implications for our understanding of specificity and diversity of coral-algal symbioses, and approaches that make use of IGVs provide the opportunity to shed light on these relationships. We have demonstrated the applicability of the metahaplotype approach for Symbiodinium clade C and in principle, it should be broadly applicable to other clades. Combining ITS2 and psbAncr amplicon sequencing analyses provides a framework whereby mixed ITS2 communities found elsewhere (for example, Sampayo et al., 2007) can be tested to identify whether monotypic communities and host specificity are found in other regions and species. This will provide a greater appreciation of the different symbiotic strategies employed by corals and help identify the environmental and biological drivers behind the single taxon and mixed community approaches.

The power of such IGV approaches is not limited to coral symbionts, and can be applied to the diverse range of microbial organisms that are currently classified by traditional ITS2 methods (for example, Schoch et al., 2012; Stern et al., 2012), particularly in metagenomics studies where IGVs could inflate diversity estimates. Our analyses demonstrate the applicability of our MED-based metahaplotype approach to characterizing relatively simple communities but it could be transferred to more complex communities. However, analysis of mixed communities would greatly benefit from the development of approaches that could identify individual metahaplotypes within a heterogeneous dataset. Transferring the metahaplotype approach to mixed communities would be a worthwhile endeavor as we clearly show that incorporating ITS2 IGVs into analyses of microbial communities reveals fundamental ecological processes that would have been, otherwise, overlooked. 
1502

a

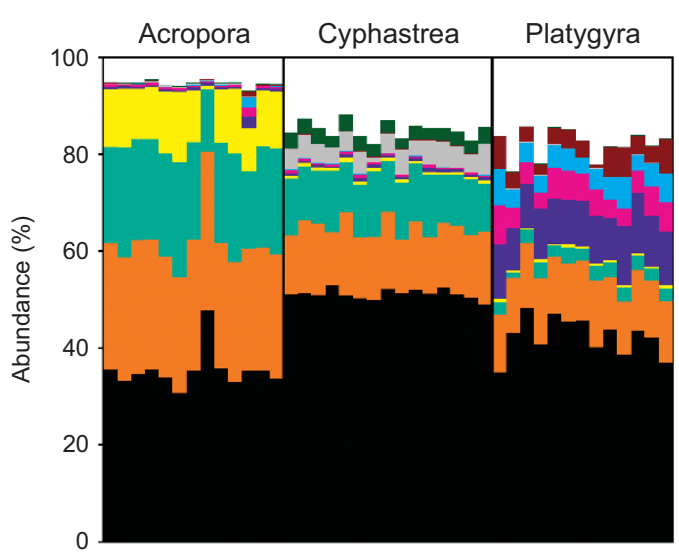

C

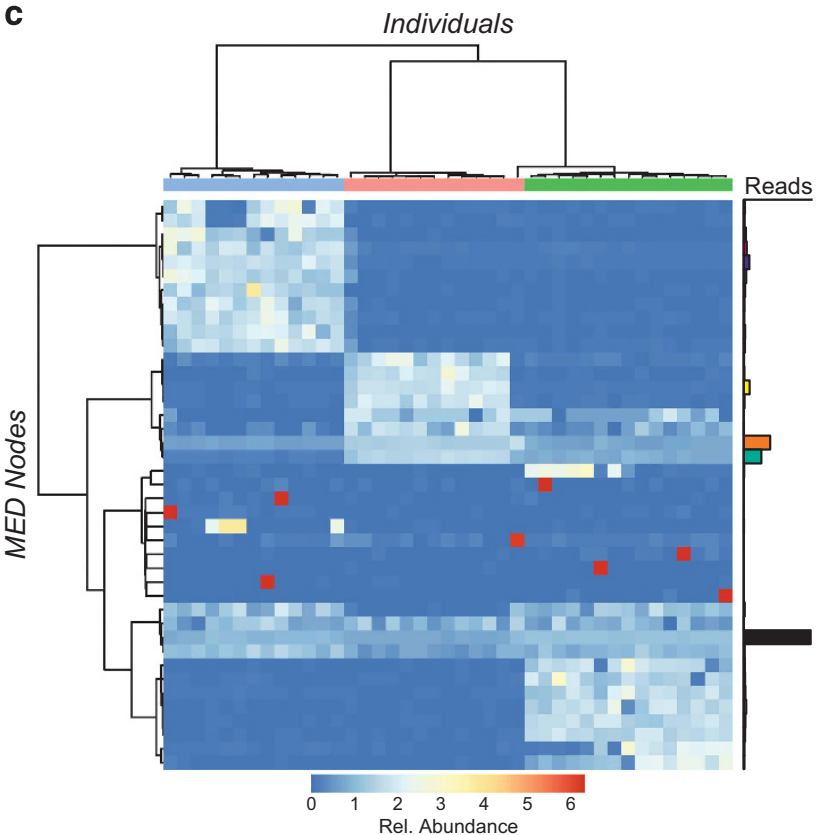

e

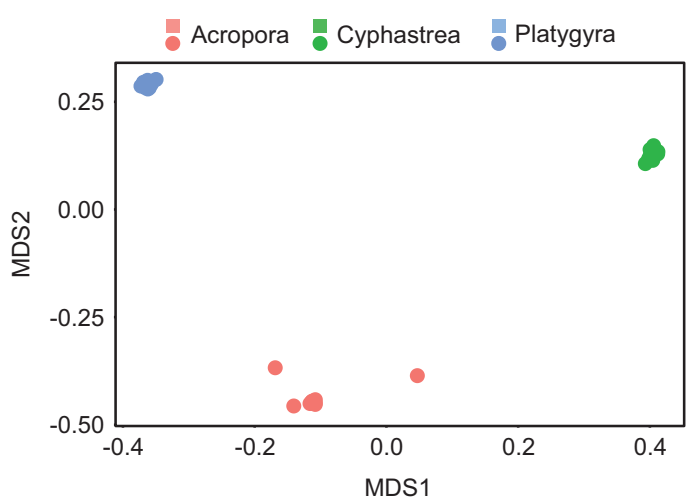

b

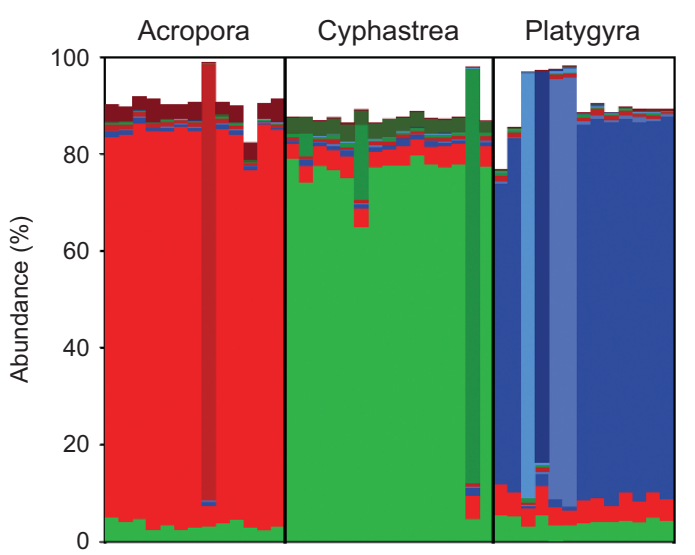

d

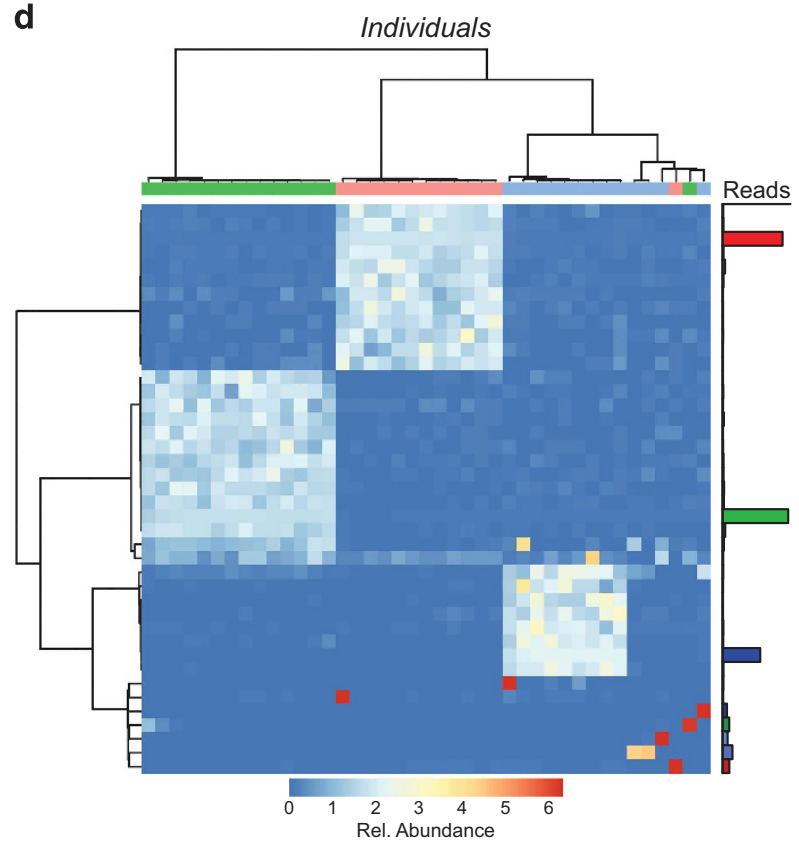

$\mathbf{f}$

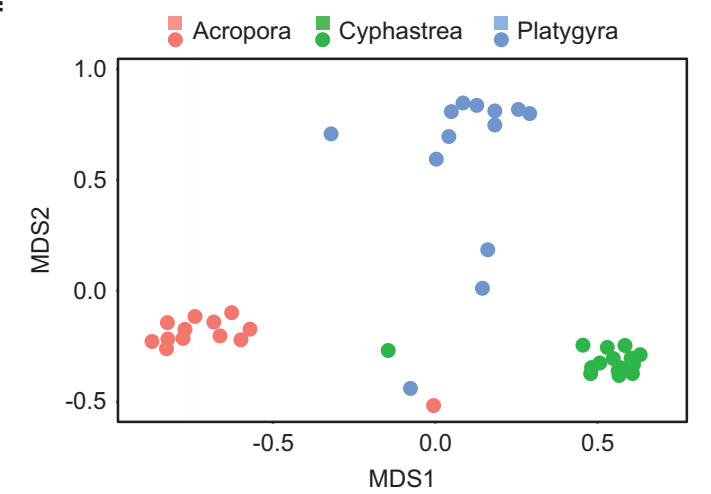

Figure 1 Symbiodinium ITS2 and psbAncr MED nodes associated with Acropora downingi, Cyphastrea microphthalma and Platygyra daedalea. (a, b) Relative composition of the 10 most abundant ITS2 (a) and psbAncr (b) MED nodes. Each colour represents a distinct MED node. See Supplementary Information for further details of ITS2 and psbAncr MED nodes. (c, d) Heat maps showing the relative proportion of MED nodes across individuals. Individuals and MED nodes are clustered based on Bray-Curtis similarity. Coloured bars indicate Acropora downingi (red), Cyphastrea microphthalma (green) and Platygyra daedalea (blue) individuals. Relative abundance of reads incorporated into each node are shown to the right of each heat map. (e, f) Bray Curtis NMDS plots for ITS2 (e) and psbAncr (f). 


\section{Conflict of Interest}

The authors declare no conflict of interest.

\section{Acknowledgements}

We thank the New York University Abu Dhabi Institute for funding and the Environment Agency Abu Dhabi for permitting. This work was supported by the New York University Core Sequencing and Bioinformatics Groups. Data used in this study are deposited in the Dryad Digital Repository. (http://dx.doi.org/10.5061/dryad.h6s54).

\section{References}

Álvarez I, Wendel JF. (2003). Ribosomal ITS sequences and plant phylogenetic inference. Mol Phylogenet Evol 29: 417-434.

Arif C, Daniels C, Bayer T, Banguera-Hinestroza E, Barbrook A, Howe CJ et al. (2014). Assessing Symbiodinium diversity in scleractinian corals via nextgeneration sequencing-based genotyping of the ITS2 rDNA region. Mol Ecol 23: 4418-4433.

Berkelmans R, Van Oppen MJ. (2006). The role of zooxanthellae in the thermal tolerance of corals: a 'nugget of hope' for coral reefs in an era of climate change. Proc R Soc B Biol Sci 273: 2305-2312.

Cunning R, Yost DM, Guarinello ML, Putnam HM, Gates RD. (2015). Variability of Symbiodinium communities in waters, sediments, and corals of thermally distinct reef pools in American Samoa. PLoS One 10: e0145099.

Eren AM, Borisy GG, Huse SM, Welch JLM. (2014). Oligotyping analysis of the human oral microbiome. Proc Natl Acad Sci USA 111: E2875-E2884.

Eren AM, Morrison HG, Lescault PJ, Reveillaud J, Vineis JH, Sogin ML. (2015). Minimum entropy decomposition: Unsupervised oligotyping for sensitive partitioning of high-throughput marker gene sequences. ISME $J$ 9: 968-979.

Hume B, D'Angelo C, Smith E, Stevens J, Burt J, Wiedenmann J. (2015). Symbiodinium thermophilum sp. nov., a thermotolerant symbiotic alga prevalent in corals of the world's hottest sea, the Persian/ Arabian Gulf. Sci Rep 5: 8562.

LaJeunesse TC. (2001). Investigating the biodiversity, ecology, and phylogeny of endosymbiotic dinoflagellates in the genus Symbiodinium using the ITS region: in search of a 'species' level marker. J Phycol 37: 866-880.

LaJeunesse TC. (2005). 'Species' radiations of symbiotic dinoflagellates in the Atlantic and Indo-Pacific since the Miocene-Pliocene transition. Mol Biol Evol 22: $570-581$.

LaJeunesse TC, Thornhill DJ. (2011). Improved resolution of reef-coral endosymbiont (Symbiodinium) species diversity, ecology, and evolution through psbA noncoding region genotyping. PLOS ONE 6: e29013.

Mai JC, Coleman AW. (1997). The internal transcribed spacer 2 exhibits a common secondary structure in green algae and flowering plants. J Mol Evol 44: 258-271.

Muscatine L, Falkowski P, Porter J, Dubinsky Z. (1984). Fate of photosynthetic fixed carbon in light-and shadeadapted colonies of the symbiotic coral Stylophora pistillata. Proc R Soc Lond B Biol Sci 222: 181-202.

Sampayo E, Franceschinis L, Hoegh-Guldberg O, Dove S. (2007). Niche partitioning of closely related symbiotic dinoflagellates. Mol Ecol 16: 3721.

Schloss PD, Westcott SL, Ryabin T, Hall JR, Hartmann M, Hollister EB et al. (2009). Introducing mothur: open-source, platform-independent, community-supported software for describing and comparing microbial communities. Appl Environ Microbiol 75: 7537-7541.

Schoch CL, Seifert KA, Huhndorf S, Robert V, Spouge JL, Levesque CA et al. (2012). Nuclear ribosomal internal transcribed spacer (ITS) region as a universal DNA barcode marker for Fungi. Proc Natl Acad Sci USA 109: 6241-6246.

Stat M, Bird CE, Pochon X, Chasqui L, Chauka LJ, Concepcion GT et al. (2011). Variation in Symbiodinium ITS2 sequence assemblages among coral colonies. PLOS ONE 6: e15854.

Stern RF, Andersen RA, Jameson I, Küpper FC, Coffroth M-A, Vaulot D et al. (2012). Evaluating the ribosomal internal transcribed spacer (ITS) as a candidate dinoflagellate barcode marker. PLoS ONE 7: e42780.

Thornhill DJ, Lajeunesse TC, Santos SR. (2007). Measuring rDNA diversity in eukaryotic microbial systems: how intragenomic variation, pseudogenes, and PCR artifacts confound biodiversity estimates. Mol Ecol 16: 53265340.

Thornhill DJ, Lewis AM, Wham DC, LaJeunesse TC. (2014). Host-specialist lineages dominate the adaptive radiation of reef coral endosymbionts. Evolution 68: $352-367$.

Supplementary Information accompanies this paper on ISME J website (http://www.nature.com/ismej) 